europäischen Entscheidungsprozesses überraschend erscheint. Auch die französische Industrie bevorzugt den ISO Standard.

Insgesamt zeigt der Fall von EMAS, daß auch die sog. neuen Instrumente der europäischen Umweltpolitik durchaus nicht frei von Implementationsproblemen sind. Wie die obigen Ausführungen verdeutlichen, können $\mathrm{zu}$ „offene“ europäische Vorgaben dazu führen, daß auf nationaler Ebene nicht genügend getan wird, um den Zielen europäischer Politik gerecht zu werden. Umgekehrt bewirken diese Defizite gleichzeitig, daß die Politikadressaten, deren Unterstïtzung eine effektive Implementation voraussetzt, ihr Interesse verlieren bzw. die Maßnahme nicht in ausreichender Weise unterstïtzen. Neue Instrumente wie EMAS sind somit nicht nur aus der regulativen, sondern auch aus der Implementationsperspektive lediglich als Ergänzung und nicht als Alternative zu traditionellen Instrumenten zu sehen.

\section{Anmerkungen}

(1) Héritier, Adrienne, Christoph Knill, Susanne Mingers: Ringing the Changes in Europe. Regulatory Competition and the Transformation of the State. Berlin 1996.

(2) Mayntz, Renate: Implementation von regulativer Politik. In: Renate Mayntz (Hrsg.), Implementation politischer Programme II, Opladen 1983, S.50-74.

(3) Lenschow, Andrea: The Implementation of EU Environmental Policy in Germany. In: Christoph Knill (Hrsg.): The Impact of National Administrative Traditions on the Implementation of EU Environmental Policy. Research Report.

Florence: European University Institute.

(4) Knill, Christoph: Appropriate Implementation. The Impact of National Administrative Traditions on European Policy-Making. In: Journal of Public Policy, Vol. 18 (1998), No.1.

(5) Knill, Christoph, Andrea Lenschow: Compliance with Europe: The Implementation of EU Environmental Policy and Administrative Traditions in Britain and Germany. Erscheint in: Journal of European Public Policy, Vol. 5 (1998), №. 4.

\section{Der Autor}

Dr. Christoph Knill ist wissenschaftlicher Mitarbeiter an der Max-Planck-Arbeitsgruppe Recht der Gemeinschaftsgüter.

Kontakt: Poppelsdorferallee 45, 53115 Bonn, Tel. $0228 / 91416-0$

Die Position der EU-Kommission zur Revision der EMAS-Verordnung

\title{
Aus den Erfahrungen lernen
}

\author{
Aus der bisherigen Praxis des EG-Öko-Audit-Systems hat sich eine Fülle von \\ Veränderungsbedarf ergeben. Basierend auf der Konsultation aller Beteiligten \\ wird die DG XI der EU-Kommission einen Revisionsvorschlag vorlegen, der dem \\ Rechnung trägt.
}

$\mathrm{D}$ e Europäische Gemeinschaft betreibt seit etwa 25 Jahren Umweltpolitik. Die bisherigen Aktionen der Gemeinschaft und der Mitgliedstaaten auf dem Gebiet des Umweltschutzes basierten fast ausschließlich auf dem Erlaß von Rechtsvorschriften (Command and Control Approach). Wir brauchen in Zukunft eine breitere Palette von Instrumenten. Dazu gehören marktorientierte Instrumente, die auf dem Prinzip der Anwendung ökonomischer und steuerlicher Anreize beruhen. Das erfolgreichste Instrument in dieser Gruppe ist das Öko-Management/ Öko-Audit-System (EMAS), das sowohl die Unterstïtzung der Unternehmen als auch die der Umweltschützer hat. EMAS ist seit 1993 in Kraft. Artikel 20 der EMAS-Verordnung sieht vor, daß die Kommission die jetzige Verordnung anhand der bei ihrer Durchfiihrung gemachten Erfahrungen überprüft und dem Rat gegebenenfalls geeignete Änderungen vorschlägt. Dies gilt insbesondere im Hinblick auf eine Ausdehnung des Systems auf andere Branchen. Dieser Prozeß läuft gerade.

\section{Grundprinzipien der Revision}

Es ist noch nicht sicher, wie der neue Vorschlag aussehen wird, einige Grundprinzipien sind jedoch bereits heute deutlich:

1. Die neue EMAS-Verordnung wird auch weiterhin ein freiwilliges Instrument sein.

2. Es wird keine Änderung der grundlegenden Bestimmungen der Verordnung geben, d.h. die teilnehmenden Unternehmen brauchen keine neuen oder andere Bestimmungen zu erfiillen, um auch in Zukunft teilnehmen zu können (Kontinuität für EMAS-registrierte Betriebe).

3. Wir werden uns auf denjenigen Gebieten enger an die ISO Normen der Reihe 14000 anlehnen, auf denen diese Normen gleich hohe Anforderungen an den Umweltschutz stellen wie EMAS. Die logischere Struktur der ISO Normen wird für gewisse Teile (Anhänge) der Verordnung übernommen werden, um eine größere Kompatibilität mit ISO zu erreichen. Dies wird zu einer Vereinfachung der Struktur des Systems führen. Ziel ist es, daß ein EMAS-registriertes Unternehmen automatisch ein ISO Zertifikat bekommt, da es alle Bedingungen für dieses Zertifikat erfüllt.

4. Die Verordnung wird auf neue Sektoren erweitert werden. Bisher können am EMASSystem alle gewerblichen Branchen teilnehmen, die in der Verordnung genannt sind. Es handelt sich im wesentlichen um das produzierende Gewerbe einschließlich Energieerzeugung und Recyclinggewerbe. In Zukunft sollen an EMAS alle Branchen teilnehmen, insbesondere auch der Dienstleistungssektor.

Neue Sektoren sind: der Handel, das Transportgewerbe, das Baugewerbe, Verteilernetze für Wasser, Gas und Strom, Land- und Forstwirtschaft, das Dienstleistungsgewerbe, Banken, Versicherungen, Krankenhäuser, Tourismus und Freizeitgewerbe, Hotels und Golfklubs und, nicht zu vergessen, die öffentliche Verwaltung.

Wahrscheinlich wird diese Ausdehnung großzügig sein und alle Branchen umfassen. Es geht dabei um die Frage: Wie muß die Öko-Audit Verordnung verändert werden, damit sie auf andere Branchen anwendbar ist? Die Definition des Standorts spielt hierbei eine besondere Rolle: wie kann man z.B. den Standort beim Transportgewerbe definieren?

\section{Vorteile für die Teilnehmer}

5. Das neue System soll einen deutlichen Mehrwert für teilnehmende Unternehmen bringen. Damit sich das EMAS-System besser am Markt durchsetzen kann, muß es attraktiv sein, insbesondere auch für kleine und mittelständische Unternehmen. Obwohl EMAS ein freiwilliges System ist, wird die Teilnahme in Zukunft für fortschrittliche Unternehmen eine Notwendigkeit sein. Experten sind überzeugt, daß EMAS eine strategische Notwendigkeit für den Unternehmenserfolg sein wird. Schon kurzfristig macht sich ein Umweltmanagementsystem bezahlt, $\mathrm{da}$ folgende Vorteile zu erwarten sind: 
Kostensenkung - hierunter fallen Einsparungen von Rohstoffen und Energie, geringere Abfallentsorgungskosten, bessere Konditionen bei Banken und Versicherungen und geringere Umwelthaftungsrisiken.

- Imagegewinn - hierunter fallen ein besseres öffentliches Erscheinungsbild als proaktives Unternehmen, Wettbewerbsvorteile, da immer mehr Kunden umweltverträglich hergestellte Produkte verlangen, eine bessere externe Kommunikation mit Anwohnern und Umweltverbänden und ein Imagegewinn bei den Mitarbeitern und damit mehr Motivation.

- Verwaltungsvereinfachung durch Erleichterungen und Vereinfachungen im Hinblick auf das bestehende Ordnungsrecht und im bürokratischen Aufwand bei Kontrollen, Dokumentationspflichten und Genehmigungsverfahren.

In einigen Bundesländern gibt es Ansätze für solche Verwaltungsvereinfachungen, aber der große Durchbruch läßt noch auf sich warten. In der neuen EMAS-Verordnung werden die Mitgliedsstaaten wahrscheinlich aufgefordert werden, solche Verwaltungsvereinfachungen einzuführen.

Die Debatte ist im Gange, und wir hoffen, daß sich bald greifbare Vorteile für EMAS-registrierte Betriebe ergeben werden.

\section{- Besonderheiten für KMU's}

6. Artikel 13 der heutigen Verordnung sieht die Möglichkeit einer besonderen Förderung der EMAS-Teilnahme von KMU's vor. Dieser Artikel wird wahrscheinlich in dem Sinne verstärkt werden, daß die Mitgliedstaaten zur Förderung der Teilnahme von KMU's aufgefordert werden.

Fast alle Interessengruppen und insbesondere die Mitgliedsstaaten, die wir konsultiert haben, waren der Meinung, daß es kein Mini-EMAS oder EMAS-light für KMU's geben darf. Die Anforderungen an die Umweltleistungen der KMU's sollten nicht reduziert werden. Damit gäbe es ein EMAS erster und zweiter Kategorie, was sicherlich nicht wünschenswert ist. Jedoch sollten bürokratische Hürden gesenkt werden, und das nicht nur für KMU's, sondern für alle Unternehmen, aber das kommt insbesondere den KMU's zugute.

\section{Weitere Veränderungen}

7. Ein weiteres Element, das die Werbung für EMAS-registrierte Betriebe und auch die Teilnahme für KMU's erleichtern wird, ist eine größere Flexibilität bei der Gestaltung der Umwelterklärung. Sie kann, wenn dies gewünscht wird, aus verschiedenen Darstellungen bestehen:

- Einem Teil, der möglichst viele Daten und Informationen enthält und in erster Linie für die Behörden bestimmt ist.

- Einem anderen Teil, der in knapper und verständlicher Form verfaßt ist, Diagramme und verständliche Darstellungen dieser Daten beinhaltet und sich insbesondere an die Öffentlichkeit richtet sowie zu Werbezwecken verwandt werden kann.

Die Umwelterklärung kann also aus verschiedenen Teilen für bestimmte Zielgruppen zusammengesetzt sein.

8. Die Kommission wird in Zusammenarbeit mit UEAPME (Europäische Union des Handwerks und der Klein- und Mittelbetriebe) einen Leitfaden über das spezifische Verbältnis von Umweltgutachtern zu KMU's und die besondere Behandlung von KMU's entwickeln.

9. Für die neue EMAS-Verordnung werden wir ferner die Möglichkeit für ein EMAS-Logo prüfen. Teilnehmende Betriebe, insbesondere KMU's, haben uns darum gebeten und würden dies sehr begrüßen. Das würde EMAS-registrierte Betriebe herausheben und die Werbung für diesen Betrieb erleichtern.

Wir hoffen, daß wir in den nächsten Monaten einen ersten Entwurf fertig haben werden, der noch 1998 von der Kommission verabschiedet und dem Rat und dem Europäischen Parlament vorgelegt werden kann. Der Rat und das Parlament brauchen für die Beratungen etwa ein bis zwei Jahre, so daß wir um die Jahrtausendwende hoffentlich eine neue EMAS-Verordnung vorliegen haben.

\section{Anmerkung}

Dieser Beitrag ist eine gekürzte Version des Beitrags von Dr. Klous Krisor in der IÖW-Schriftenreihe 125/97 "ÖkoAudit in kleinen und mittleren Unternehmen".

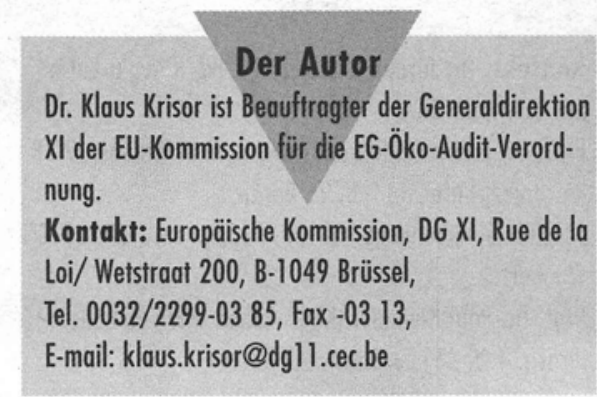

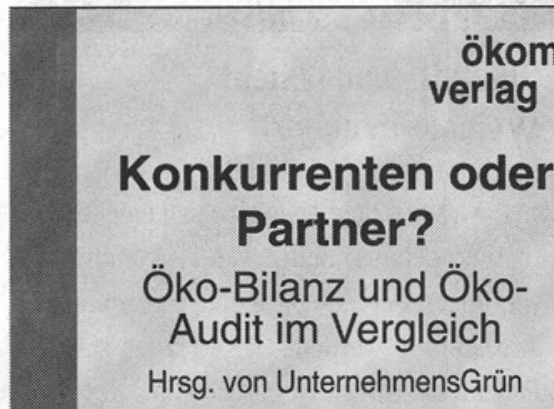

Nachhaltige Entwicklung ist ohne vergleichende Analyse nicht denkbar. Ökobilanzen liefern hierfür wichtige Grundlagen: Daten und Kennziffern, mit deren Hilfe Produkte und Produktionsverfahren umweltfreundlicher gestaltet werden können.

Welchen Stellenwert besitzt die Ökobilanz angesichts moderner Umweltmanagementkonzepte wie z.B. dem Öko-Audit? Wie effektiv sind Ökobilanzen und Öko-Audit im Vergleich? Stehen sie in Konkurrenz zueinander oder gibt es sinnvolle Ergänzungen?

Edith Memel: Die ökologische Ausrichtung unseres Wirtschaftssystems (Ökobilanzen, Öko-Audit und Ökosteuer). Helge Majer: Das Spannungsverhältnis von Weltwirtschaft und Region - Rudolf Hickel: Der ökologische Umbau aus volkswirtschaftlicher Sicht - Bernd Wagner: Sustainable Development aus Sicht des einzelnen Unternehmens . Mario Schmidt: Öko-Audit versus Öko-Bilanz. Konkurrenz oder sinnvolle Ergänzung? . Martin Grammer: Produkt-Ökobilanz Erfahrungen bei der Grammer AG . Helmut Lübke: Standort-Ökobilanz - Erfahrungen bei COR Wohn- und Büromöbel H. Lübke GmbH \& Co - Jeanette A. Böning: Unternehmens-Ökobilanz Erfahrungen bei der Allianz-Versicherung AG - Streitgespräch zwischen Krista Sager (Bündnis 90/Grüne) und Wolfgang Ritter (BDI): Wie wird die Wirtschaft ökologisch? · Unternehmensgrün Thesenpapiere: Öko-Audit, Öko-Bilanzen, ÖkoSteuer

UnternehmensGrün $\bullet$ ökom verlag Band 3 : Waltherstr. 29, 119 Seiten. 80337 München, DM 19,80 (zzgl. : Tel. (089) 544 184-0, Versandkosten) - Fax (089) 544 184-99 
(c) 20I0 Authors; licensee IÖW and oekom verlag. This is an article distributed under the terms of the Creative Commons Attribution Non-Commercial No Derivates License (http://creativecommons.org/licenses/by-nc-nd/3.o/), which permits unrestricted use, distribution, and reproduction in any medium, provided the original work is properly cited. 